\title{
Caracterização do fármaco hipoglicemiante glibenclamida
}

\author{
Christiane Gino Colu Nery, Maria Arlete da Silva Pires, Gerson Antônio Pianetti, \\ Cristina Duarte Vianna-Soares*
}

\begin{abstract}
Divisão de Desenvolvimento Farmacotécnico e Biotecnológico, Fundação Ezequiel Dias/FUNED, Departamento de Produtos Farmacêuticos, Universidade Federal de Minas Gerais
\end{abstract}

*Correpondência:

C. D. Vianna-Soares

Departamento de Produtos

Farmacêuticos

Universidade Federal de Minas Gerais

Av. Antônio Carlos, 6627

31270-901 - Belo Horizonte - MG,

Brasil

E-mail: cviannas@ufmg.br
A glibenclamida (GLIB) é um fármaco de segunda geração, administrado por via oral na forma de comprimidos, utilizado para o tratamento de Diabetes mellitus. GLIB possui baixa solubilidade aquosa, o que pode levar a uma baixa liberação a partir de formas farmacêuticas sólidas no teste de dissolução e, portanto, a variabilidades no tratamento. Neste estudo, avaliam-se as características da matéria-prima GLIB, que podem influenciar o perfil de dissolução, e conseqüentemente, a biodisponibilidade, por meio de técnicas tais como, adsorção de nitrogênio, difração de raio laser, análise térmica, espectroscopia por IV/UV e difração de raios $X$.

\author{
Uniterms \\ - Agente hipoglicemiante \\ - Glibenclamida/ \\ caracterização
}

\section{INTRODUÇÃO}

Os primeiros registros de genéricos hipoglicemiantes, contendo cloridrato de metformina e produzidos pelas empresas Biosintética e Merck, foram autorizados pela Agência Nacional de Vigilância Sanitária (ANVISA) em julho de 2001 (Brasil, 2001a). No caso de medicamento genérico de glibenclamida (GLIB), o registro concedido à Biosintética, impactou em 35\% de economia (Brasil, 2001b) para o consumidor quando comparado ao valor praticado pelo medicamento referência Daonil ${ }^{\circledR}$ (Aventis Pharma Ltda.).

Considerando que o acompanhamento da saúde do diabético, que é uma das prioridades da Anvisa, é responsabilidade do município e o atendimento mais especializado é função do estado (Brasil, 2001c) a Fundação Ezequiel Dias (FUNED, Belo Horizonte, MG) laboratório oficial do estado de Minas Gerais produz o medicamento GLIB $5 \mathrm{mg}$ comprimidos, alvo do presente trabalho.
Em estudos prévios, Babu e Pandit (1995) verificaram variação acentuada na velocidade de liberação de GLIB nos testes in vitro (de dissolução) e, conseqüentemente, na biodisponibilidade do produto a partir de diferentes fabricantes de comprimidos do fármaco. No mercado internacional há comprimidos produzidos com tamanhos variados de partículas da matéria-prima GLIB, a qual pode ser micronizada ou microfina e não micronizada ou comum. No entanto, as faixas de especificação para granulometria não são bem definidas na literatura. Verifica-se que as formulações produzidas a partir de diferentes tamanhos de partículas de GLIB matérias-primas não são uniformemente bioequivalentes e a dose deve ser ajustada individualmente no caso de alteração de tipos de prescrições (AHFS, 2000). Soma-se a isto, a necessidade de desenvolver um método de dissolução para avaliação in vitro (Nery et al. 2007), ausente na monografia de GLIB comprimidos de vários compêndios como as Farmacopéias Brasileira (Farmacopéia, 2001), Americana (United, 2005), Mexica- 
na (Farmacopea, 2000), Japonesa (Japanese, 2003) e Britânica (British, 2003b).

Desta forma, é iminente a necessidade de rever e aprimorar a especificação ou caracterização da GLIB, uma vez que, só os testes farmacopéicos atualmente exigidos, não são suficientes para apontar diferenças sutis entre as matérias-primas. Esta avaliação pode ser uma das ferramentas da qualidade da referida matéria-prima que, mesmo se originada de diversos fabricantes e utilizada na fabricação de comprimidos, deve proporcionar discriminação de resultados ou interpretação consistente.

A glibenclamida (I, 494,01 g/mol, $\mathrm{C}_{23} \mathrm{H}_{28} \mathrm{ClN}_{3} \mathrm{O}_{5} \mathrm{~S}$ ) é um pó cristalino branco ou quase branco, inodoro praticamente sem sabor (Merck, 2001; Takla, 1981; Clarke, 1986). Apresenta pKa igual a 5,3, característico de um ácido fraco, coeficiente de partição (Kassim et al., 2004) óleo/água (log P) igual a 3,53 e, baixo valor de absorvância na região do ultravioleta, $300 \mathrm{~nm}$, em metanol (A1\%,1 cm=63) e em solução metanólica acidificada (Clarke, 1986). A GLIB é solúvel em soluções de hidróxidos alcalinos e dimetilformamida, ligeiramente solúvel em diclorometano e clorofórmio $(1: 36 \mathrm{p} / \mathrm{V})$, pouco solúvel em etanol $(1: 330 \mathrm{p} / \mathrm{V})$ e metanol $(1: 250 \mathrm{p} / \mathrm{V}) \mathrm{e}$ insolúvel em água e éter etílico. As faixas de fusão descritas (Farmacopéia, 2001; Merck, 2001; TAKLA, 1981; Clarke, 1986; British, 2003a; European, 1997) são: $172-174^{\circ} \mathrm{C}$; 169 $170{ }^{\circ} \mathrm{C}, 168-170{ }^{\circ} \mathrm{C}$ e $169-174{ }^{\circ} \mathrm{C}$. Duas impurezas da matéria-prima GLIB, apresentadas pela Farmacopéia Britânica (2003a) são quimicamente denominadas 4-[2-(5-cloro-2metoxibenzamido) etil] benzenossulfonamida(II) e N-4[2-(5cloro-2-metoxibenzamido) etil] benzenossulfonil-Nmetilcarbamato de etila(III).

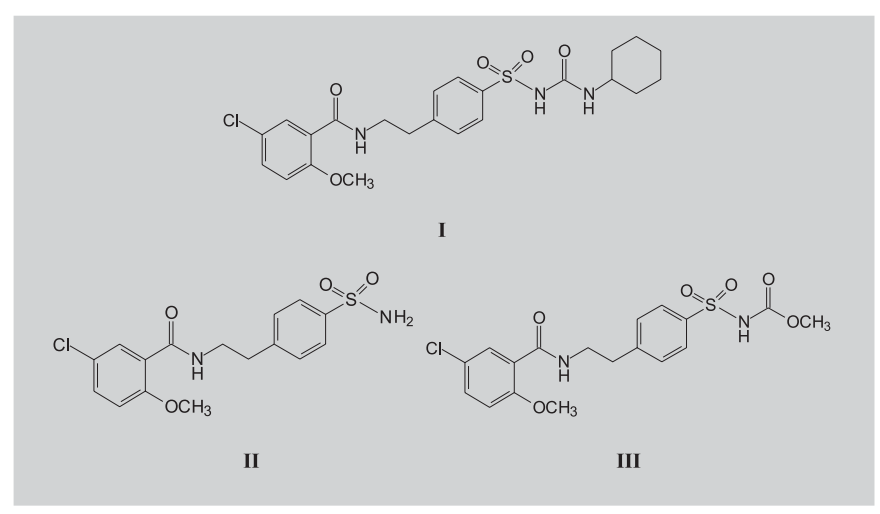

Vários autores descrevem que a baixa biodisponibilidade da GLIB está relacionada ao fato de ser insolúvel em água e, conseqüentemente, apresentar velocidades reduzidas de liberação nos testes in vitro de dissolução (Babu, Pandit, 1995; Ghosh et al., 1998; Hassan, Najib, Suleiman, 1991; Suleiman, Nagib, 1989).
Inúmeros esforços, em todo o mundo, têm sido conduzidos na tentativa de aumentar a solubilidade e melhorar a biodisponibilidade de fármacos com características semelhantes à GLIB. Recursos técnicos como a micronização, a dispersão molecular, a incorporação de surfactantes, a complexação de inclusão em ciclodextrina e a transformação da fase sólida em formas polimorfas ou amorfas têm sido empregadas no caso da GLIB (Ghosh et al., 1998; Hassan, Najib, Suleiman, 1991; Suleiman, Najib, 1989).

A compreensão da natureza polimorfa de um fármaco ou excipiente é de fundamental importância para o delineamento das formulações farmacêuticas. $\mathrm{O}$ fármaco pode sofrer alterações da forma cristalina durante processos como, por exemplo, secagem do granulado, moagem ou compactação. A mudança da forma cristalina pode acarretar alterações na temperatura de fusão, densidade, solubilidade, estabilidade física ou química, dissolução e biodisponibilidade de um fármaco e de sua formulação (Clas, Dalton, Hancock, 1999; Yoshihashi et al., 2000). Por isso, é importante a caracterização por técnicas diversas tais como, análise térmica, determinações do tamanho da partícula e da área superficial, espectrométricas (UV e IV), difração de raios X, e outras.

Para a GLIB, encontram-se descritos dois polimorfos I e II, e dois pseudopolimorfos obtidos a partir da cristalização da forma I em diferentes solventes. A forma mais estável I é aparentemente menos solúvel e tem reduzida dissolução (Suleiman, Najib, 1989). A um terceiro polimorfo, obtido a partir da tentativa de elucidação da transição vítrea pelo aquecimento, resfriamento e reaquecimento da GLIB, foram atribuídos baixos valores de dissolução e biodisponibilidade da GLIB em comprimidos (Panagopoulou-Kaplani, Malamataris, 2000). Maiores porcentagens de liberação de GLIB amorfa foram obtidas quando comparadas às porcentagens de GLIB cristalina ou do produto comercial DaonilÒ após estudos de dissolução (Hassan, Najib, Suleiman, 1991).

A averiguação de tamanho das partículas e sua distribuição do fármaco é pré-requisito fundamental para muitas operações de produção e processamento que envolvem materiais na forma de pó. Partículas esféricas apresentam um comportamento previsível por modelos relativamente simples. Desvios da forma esférica ou, formas difíceis de serem descritas por figuras geométricas simples (Lowel, Shields, 1991) têm um desempenho mais difícil de ser previsto.

Neste estudo pretende-se caracterizar diferentes matérias-primas GLIB existentes no mercado brasileiro local quanto à sua descrição microscópica óptica e eletrônica, faixa de fusão, densidade compactada, massa e área superficial específicas, características termoanalíticas e 
espectroscópicas, bem como à difração da luz monocromática de raios $\mathrm{X}$ e distribuição granulométrica. Isto permitirá avaliar o impacto de diferentes matérias-primas GLIB utilizadas na fabricação de comprimidos, a fim de melhorar a liberação do fármaco in vitro, e conseqüentemente in vivo.

\section{PARTE EXPERIMENTAL}

\section{Material}

GLIB substância química de referência da Farmacopéia Brasileira (SQRFB, lote 1018, 99,96\% pureza) adquirida do Instituto Nacional de Controle de Qualidade em Saúde (INCQS, Rio de Janeiro, RJ).

Várias amostras de matéria-prima GLIB (procedentes da Índia) foram gentilmente cedidas por fornecedores do mercado brasileiro. Para fins do estudo proposto, as amostras enviadas foram agrupadas pela semelhança quanto à característica de distribuição granulométrica e identificadas como MP1, MP2, MP3 e MP4 sendo MP2 e MP3 provenientes do mesmo fabricante.

Os equipamentos utilizados foram: analisador de densidade compactada (Vankel); analisador de massa específica e de de área superficial específica NOVA 1200 (Quantachrome); analisador de partículas por difração de laser LS13320 com módulo universal para líquidos e faixa de leitura de 0,040 a $2000 \mathrm{~mm}$ (Beckman Coulter); balança semi-analítica BG 440, precisão 0,01g (Gehaka); balança analítica AT 201, precisão 0,01 mg (Metler Toledo); calorímetro de varredura exploratória $822 \mathrm{e} / 700 \mathrm{com}$ sistema de resfriamento e amostrador automático com perfuração de tampa (Metler Toledo); analisador termogravimétrico TGA/SDTA 851 (Metler Toledo); difratômetro de raios X PW3710 (Philips); equipamento para determinação de ponto de fusão 9100 (Electrothermal); espectrofotômetro infravermelho FT-IR Spectrum One (Perkin-Elmer; espectrofotômetro UV-3 (Unican); estufa 306/3 (Fanem); microscópio óptico estereoscópico DX 50 acoplado à câmera de vídeo e impressora específica para captura de sinal (Olympus); microscópio eletrônico de varredura 5410 (ISM).

\section{Métodos}

\section{Descrição}

As matérias-primas foram visualizadas a olho nu para verificação do aspecto e da cor e, por microscopias óptica (MO) e eletrônica de varredura (MEV) para avaliação do tamanho e forma dos cristais. Em MO, o material foi preparado misturando-se pequena quantidade da maté- ria-prima em 1 gota de água desionizada. Colocou-se a lamínula sobre a mistura e o material foi avaliado utilizando-se aumento de 400 vezes. Em MEV, a matéria-prima foi submetida à metalização com ouro para tornar a superfície condutora, e colocada em um porta-amostra para avaliação com aumentos de 750 e 1500 vezes.

\section{Faixa de fusão}

A determinação da faixa de fusão das matérias-primas $(n=3)$ foi realizada seguindo o método geral V.2.2, Farmacopéia Brasileira quarta edição (Farmacopéia, 1988)

\section{Densidade compactada}

Pesaram-se, analiticamente, $15 \mathrm{~g}$ da matéria-prima $(n=2)$ que foram transferidos para as duas provetas calibradas do equipamento. As provetas foram submetidas a batidas seqüenciais e o valor da densidade compactada $(\mathrm{g} /$ $\mathrm{mL}$ ) foi determinado dividindo-se a massa da amostra pelo valor médio dos volumes finais encontrados.

\section{Massa e área superficial específicas}

Determinaram-se a massa específica (ME) e a área superficial específica (ASE) para as matérias-primas introduzidas em picnômetro apropriado acoplado ao analisador de massa e área superficial específicas por meio da isoterma do método Brunauer, Emmett, Teller (BET) utilizando adsorção por gás nitrogênio (Brunauer, Emmett, Teller, 1938).

\section{Termogravimetria com analisador térmico diferencial acoplado (TG/DTA)}

A variação representativa de massa (perda ou ganho) de GLIB matéria-prima MP2 em função da temperatura foi avaliada por um único método na faixa de temperatura compreendida entre

35 e $400{ }^{\circ} \mathrm{C}$ e fluxo de aquecimento $10^{\circ} \mathrm{C} / \mathrm{min}$. Pesaram-se, analiticamente, cerca de $10 \mathrm{mg}$ da matéria-prima em cadinho de alumínio (capacidade $70 \mathrm{~mL}$ ), previamente tarado. Não houve pré-tratamento da amostra e a análise ocorreu sob pressão atmosférica e gás nitrogênio com fluxo $50 \mathrm{~mL} / \mathrm{min}$.

\section{Calorimetria Exploratória Diferencial (DSC)}

A técnica termoanalítica por meio de DSC foi utilizada para caracterização das propriedades térmicas da matéria-prima GLIB no que se refere à determinação do ponto de fusão, transição vítrea, calor específico, avaliação da presença ou não de polimorfos. A programação de vários métodos utilizados quanto à faixa de temperatura $\mathrm{e}$ 
fluxo de aquecimento e/ou resfriamento, foram: 50 a 320 ${ }^{\circ} \mathrm{C}, 5^{\circ} \mathrm{C} / \mathrm{min}$ (método 1 ); 25 a $180^{\circ} \mathrm{C}, 20^{\circ} \mathrm{C} / \mathrm{min}$; isoterma a $180^{\circ} \mathrm{C}$ por 1 minuto; de $180 \mathrm{a}-10^{\circ} \mathrm{C} ; 30^{\circ} \mathrm{C} / \mathrm{min}$; de -10 a $190{ }^{\circ} \mathrm{C} ; 20^{\circ} \mathrm{C} / \mathrm{min}$ (método 2); 25 a $180{ }^{\circ} \mathrm{C}, 1{ }^{\circ} \mathrm{C} / \mathrm{min}$ (método 3 ).

Cerca de 1,5 a 3,0 mg de GLIB SQRFB e das matérias-primas MP1, MP2, MP3 e MP4, exatamente pesados, foram separadamente transferidos para cadinhos (capacidade $40 \mathrm{~mL}$ ) com tampas perfuradas. As condições de atmosfera e gás de arraste foram idênticas às descritas em TG/DTA.

\section{Espectrofotometria na região do infravermelho (IV)}

Os espectros das matérias-primas (em pó, previamente dessecadas) na região do infravermelho (reflectância) foram traçados na faixa entre $4000 \mathrm{~cm}^{-1} \mathrm{e}$ $650 \mathrm{~cm}^{-1}$.

\section{Espectrofotometria na região do ultravioleta}

Os espectros das soluções padrão e das matérias-primas em solução metanólica acidificada $(0,1 \mathrm{mg} / \mathrm{mL}, \mathrm{n}=3)$ foram traçados na região do ultravioleta na faixa entre 200 a $350 \mathrm{~nm}$ adaptando-se a técnica de identificação, constante na monografia de GLIB, descrita na Farmacopéia Brasileira quarta edição (Farmacopéia, 2001).

\section{Difração de raios $\mathrm{X}$}

Os espectros de difração de raios $\mathrm{X}$ das matériasprimas foram obtidos empregando-se um sistema monocromador em cristal de grafite, radiação $\mathrm{Cu}-\mathrm{Ka}$ com comprimento de onda (1) igual a $1,54186 \mathrm{~nm}$, voltagem e corrente iguais a $40 \mathrm{kV}$ e $20 \mathrm{~mA}$, respectivamente e, varredura de raios $\mathrm{X}$ de ângulo aberto $2 \mathrm{q}$ entre $0^{\circ}$ e $80^{\circ}$.

Os espectros obtidos foram confrontados com o depositado em biblioteca do equipamento (cartão JCPDS) em que a GLIB possui estrutura cristalina com sistema do tipo monoclínico. Neste tipo de sistema, o tamanho dos lados da cela unitária, denominados $a, b$ e $c$ são diferentes entre si. Os ângulos a (entre os lados $b$ e $c$ ) e g (entre os lados $a$ e $b$ ) são de $90^{\circ}$ e diferentes do ângulo b (entre os lados $a \mathrm{e} c$ ) (Florence, Attwood, 2003).

\section{Análise de distribuição granulométrica}

O modelo óptico de Fraunhofer foi utilizado para avaliar a distribuição granulométrica das matérias-primas em suspensão na presença de detergente (comercial) até obtenção de um obscurecimento entre 8 e $12 \%$.

\section{RESULTADOS E DISCUSSÃO}

\section{Descrição}

A olho nu, todas as matérias-primas apresentaram-se como pó fino de cor branca com grande tendência à aglomeração, à exceção da amostra MP1 que, nitidamente, era constituída por cristais maiores com boa fluidez.

A microscopia óptica obtida para as matérias-primas MP1, MP2, MP3 e MP4 está ilustrada na Figura 1. A matéria-prima MP1 (a) apresentou cristais grandes e uniformes. Pode-se observar cristais irregulares e de tamanhos variados em cada uma das amostras das matérias-primas MP2 (b), MP3 (c) e MP4 (d), caracterizando heterogeneidade.

A microscopia eletrônica de varredura das matériasprimas MP1, MP2, MP3 e MP4 está apresentada na Figura 2. A matéria-prima MP1 (a) apresentou cristais grandes com visível porosidade (macroporos, poros com dimensões $>500 \AA$ ou $>50 \mathrm{~nm} \mathrm{ou}>0,05 \mathrm{~mm}$ ) diferindo muito, em tamanho e forma, das demais. As matérias-primas MP2 (b), MP3 (c) e MP4 (d) apresentam-se como policristais mais compactos e formando agregados, confirmando a tendência à aglomeração.

\section{Faixa de fusão}

As faixas de fusão encontradas na literatura para a matéria-prima GLIB foram: $172-174{ }^{\circ} \mathrm{C}$ (Clarke, 1989); $169-170{ }^{\circ} \mathrm{C}, 168-170{ }^{\circ} \mathrm{C}$ (Takla, 1981) e $169-174{ }^{\circ} \mathrm{C}$ (Farmacopéia, 2001; British, 2003a; European, 1997). Os resultados da faixa de fusão das matérias-primas analisadas estão apresentados na Tabela I. Os valores obtidos, na faixa entre 169 e $171^{\circ} \mathrm{C}$, estão de acordo com os da literatura.

\section{Análise Térmica}

A curva TG obtida para a matéria-prima MP2 está apresentada na Figura 3a. Observaram-se três perdas de massa ao longo da análise: a primeira curva iniciou-se em $176^{\circ} \mathrm{C}$ e terminou em $279^{\circ} \mathrm{C}$ resultando em uma perda de $22,77 \%$ da massa inicial; a segunda iniciou-se em $282^{\circ} \mathrm{C}$ e terminou em $347^{\circ} \mathrm{C}$ gerando uma perda de $11,42 \%$; a terceira, e última, iniciou-se em $347^{\circ} \mathrm{C}$ e terminou em $387^{\circ} \mathrm{C}$ com perda de $33,92 \%$. O resíduo final foi de $31,68 \%$ da massa inicial, ou seja, 3,69 mg. A derivada da curva TG (DTG), Figura 3b, resultou em uma curva com um pico bem alargado referente à primeira perda de massa, um ombro referente à segunda e um pico mais acentuado referente à terceira perda de massa. A curva de análise térmica diferencial (DTA, Figura 3c) sinalizou uma variação de temperatura referente à fusão do material iniciando-se em $172{ }^{\circ} \mathrm{C}$ com pico em $177^{\circ} \mathrm{C}$. 


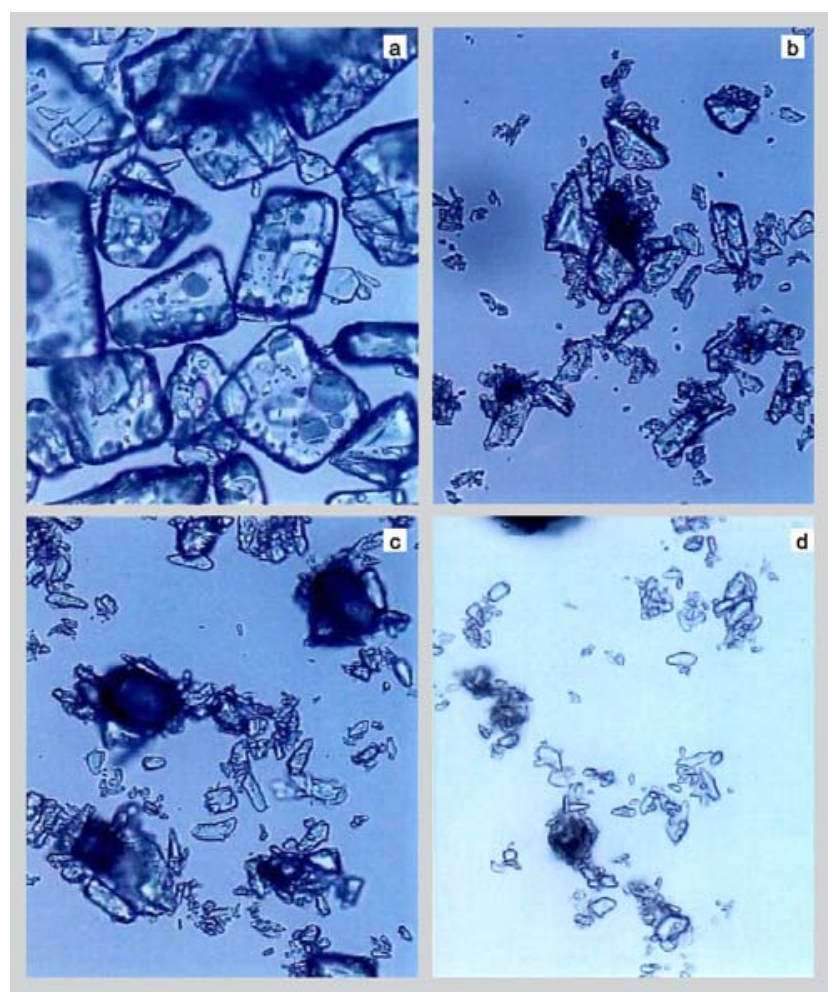

FIGURA 1 - Microscopia óptica de GLIB matérias-primas MP1 (a), MP2 (b), MP3 (c) e MP4(d) dispersas em água, aumento 400X.

As curvas DSC de GLIB SQRFB e da matéria-prima MP4, representativa das demais, obtidas pelo método 1 estão apresentadas na Figura 4a,b, respectivamente. Em ambas, observou-se somente um evento endotérmico referente à fusão da matéria-prima iniciando-se aproximadamente em

$173^{\circ} \mathrm{C}$. Após a fusão, a linha de base só voltou ao patamar inicial depois de $250^{\circ} \mathrm{C}$ aproximadamente. Este fato pode estar relacionado com a primeira perda de massa sinalizada pela curva TG. Ressalta-se, entretanto, que não há uma correlação exata entre as temperaturas destas

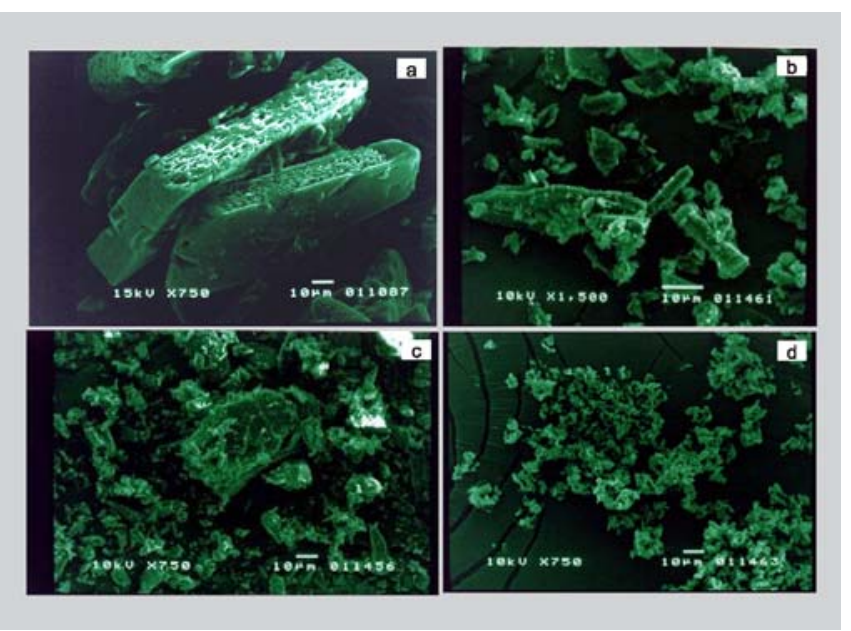

FIGURA 2 - Microscopia eletrônica de varredura de GLIB matérias-primas MP1 (a, 750X), MP2 (b, 1500X,) MP3 (c, 750X) e MP4 (d, 750X), $10 \mathrm{kV}$.

curvas e a de TG porque foram utilizadas razões de aquecimento diferentes. A partir dos resultados da aplicação do método 1, o método 2 foi delineado com o objetivo de investigar em qual temperatura ocorreria a transição vítrea (Tg) para a GLIB no estado amorfo. Hassan e colaboradores (1991) relataram um valor de Tg, se existente, igual a $71^{\circ} \mathrm{C}$ para determinada matéria-prima GLIB (pico de fusão $174,4{ }^{\circ} \mathrm{C}$ ) aquecida a $185^{\circ} \mathrm{C}$ e resfriada, rapidamente, em banho de gelo com agitação. Na curva DSC, Figura 5, obtida pelo método 2 para a matéria-prima MP2, representativa das demais, observou-se um pico característico da fusão da GLIB iniciado em $173{ }^{\circ} \mathrm{C}$, seguido pela linha do resfriamento. Não se observou evento exotérmico referente a uma possível recristalização indicando que o material assumiu a forma totalmente amorfa. Este fato pode ser comprovado pela $\mathrm{Tg}$ em $61{ }^{\circ} \mathrm{C}$ na segunda rampa de aquecimento do método e, pela ausência do evento endotérmico (característico da fusão dos cristais de GLIB), na última etapa do processo. O méto-

TABELA I - Resultados de fusão obtidos visualmente e por DSC ${ }^{\mathrm{b}}$ e para a SQRFB e matérias-primas GLIB.

\begin{tabular}{lcccc}
\hline Substância & Faixa de fusão $\left({ }^{\circ} \mathrm{C}\right)$ & Massa $^{\mathrm{b}}(\mathrm{mg})$ & Entalpia, $^{\mathrm{D}} \mathrm{DH}^{\mathrm{b}}(\mathrm{J} / \mathrm{g})$ & $\mathrm{T}_{\text {onset }}{ }^{\mathrm{b}}\left({ }^{\circ} \mathrm{C}\right)$ \\
\hline GLIB SQRFB & $169,7-171,8$ & 1,99 & 100 & 170 \\
MP 1 & $169,3-171,3$ & 1,96 & 97 & 170 \\
MP 2 & $169,2-170,1$ & 2,01 & 110 & 170 \\
MP 3 & $169,2-169,7$ & 1,96 & 97 & 169 \\
MP 4 & $169,2-169,8$ & 2,08 & 110 & 171 \\
\hline
\end{tabular}

a obtido visualmente pelo método do capilar; ${ }^{\mathrm{b}}$ condições: cadinho de alumínio $40 \mathrm{~mL}$ com tampa perfurada; pressão atmosférica com fluxo do gás nitrogênio $50 \mathrm{~mL} / \mathrm{min}$; faixa de temperatura 25 a $180^{\circ} \mathrm{C}$ com razão de aquecimento de 1 ${ }^{\circ} \mathrm{C} / \mathrm{min}$. Pico de fusão para todas as substâncias, $171^{\circ} \mathrm{C}$. 
do 2 exemplifica o modo como ocorre a decomposição térmica do material.

Para a determinação da temperatura inicial de fusão $\left(\mathrm{T}_{\text {onset }}\right)$ e da quantificação do calor envolvido neste processo (entalpia de fusão, DH) aplicou-se o método 3 para a SQRFB e matérias-primas GLIB utilizando-se razão de aquecimento bem lenta. Os resultados obtidos a partir do método 3 estão compilados na Tabela I.

Os valores de $T_{\text {onset }}$ foram muito próximos para GLIB SQRFB e matérias-primas. O valor de DH apresentou uma pequena oscilação, entre 97 e $110 \mathrm{~J} / \mathrm{g}$ sugerindo tratar-se de estruturas cristalinas semelhantes. No entanto, ressalta-se que o cálculo de DH fica comprometido tanto para a GLIB SQRFB quanto para as matérias-primas uma vez que, a linha de base não retornou ao seu valor inicial após a fusão. Neste caso, o emprego de um outro tipo de linha de base, como a sigmoidal (CLAS, 1999) poderia trazer resultados mais bem definidos. Em nenhum dos métodos empregados observaram-se eventos indicativos da presença de polimorfos ou pseudopolimorfos nas matérias-primas analisadas.

\section{Espectrofotometria na região do infravermelho}

Segundo Takla (1981) as principais bandas para GLIB são 1724, 1613, 1515, 1471, 1333 e $1163 \mathrm{~cm}^{-1}$; as bandas em 3363 e $3313 \mathrm{~cm}^{-1}$ são devido ao estiramento N$\mathrm{H}$ da uréia; a banda em $1515 \mathrm{~cm}^{-1}$ refere-se à amida da uréia; as bandas em $1333 \mathrm{~cm}^{-1} \mathrm{e} 1163 \mathrm{~cm}^{-1}$ referem-se ao $\mathrm{SO}_{2}-\mathrm{N}$ e a banda duplicada de $-\mathrm{SO}_{2}$, característica das sulfoniluréias, respectivamente. Clarke (1986) descreve como máximos característicos apenas as bandas em 1718, $1623,1524,1276,1160$, e $823 \mathrm{~cm}^{-1}$.

Os valores máximos encontrados para GLIB SQRFB e matérias-primas, próximos àqueles relatados na literatura citada foram 3366, 3312, 1713, 1615, 1478, 1340, 1277, 1156 e $819 \mathrm{~cm}^{-1}$. O espectro infravermelho representativo para GLIB SQRFB e para a matéria-prima MP4, encontrase na Figura 6a.

\section{Espectrofotometria na região do ultravioleta}

Segundo a monografia da GLIB na Farmacopéia Brasileira quarta edição (2001), o espectro ultravioleta das soluções de GLIB SQRFB e de matérias-primas a $0,1 \mathrm{mg} /$ $\mathrm{mL}$, em solução metanólica acidificada (ácido clorídrico 0,5 mol/L:metanol, 1:49 V/V), deve exibir máximos de absorvância em 300 e $275 \mathrm{~nm}$. As absorvâncias em $300 \mathrm{~nm}$ e $275 \mathrm{~nm}$ devem estar compreendidas entre 0,61 e 0,65 e, entre 0,27 e 0,32 , respectivamente.

O espectro representativo das soluções de GLIB SQRFB e de matérias-primas (Figura 6b) exibiu máximos de absorção em 275 e $300 \mathrm{~nm}$ e seus valores de absorvância em triplicata (Tabela II) estão de acordo com a faixa especificada.

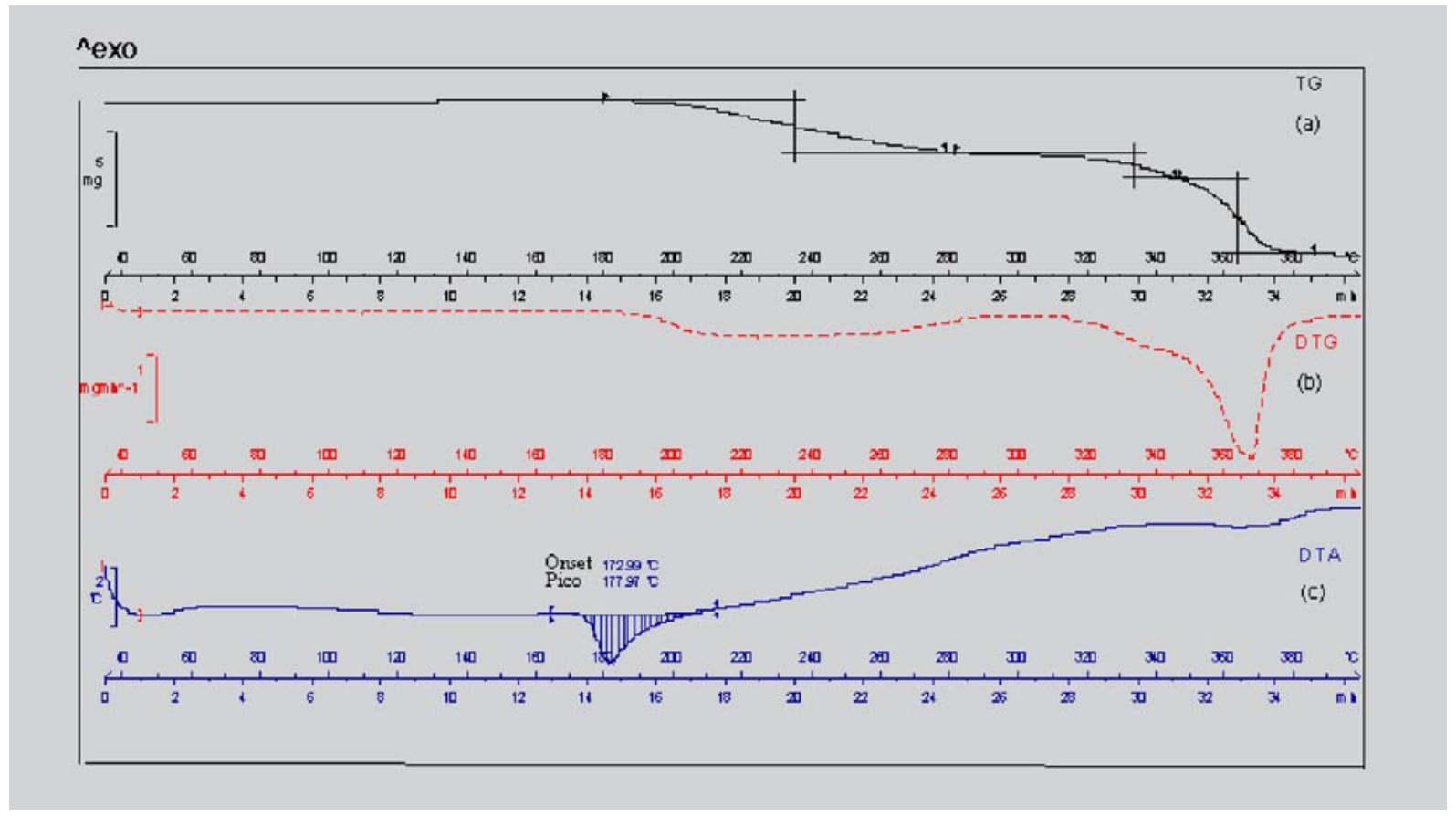

FIGURA 3 - Curvas TG (a), DTG (b) e DTA(c) para GLIB matéria-prima MP2. Condições: cadinho de alumínio $70 \mathrm{~mL}$; pressão atmosférica com fluxo do gás nitrogênio $50 \mathrm{~mL} / \mathrm{min}$; razão de aquecimento $10^{\circ} \mathrm{C} / \mathrm{min}, 35-400{ }^{\circ} \mathrm{C}$. 


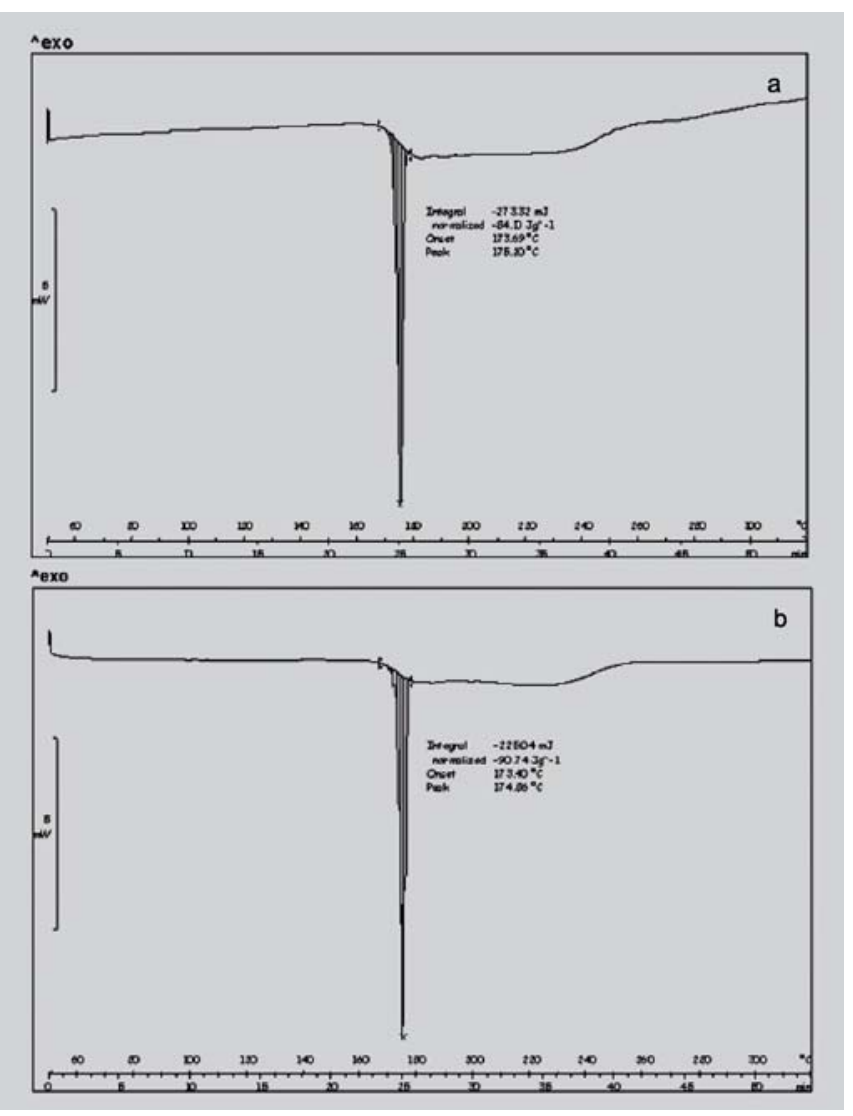

FIGURA 4 - Curvas DSC de GLIB SQRFB (a) e da matériaprima MP4 (b) obtidas pelo método 1. Condições: cadinho de alumínio $40 \mathrm{~mL}$ com tampa perfurada; pressão atmosférica com fluxo do gás nitrogênio $50 \mathrm{~mL} / \mathrm{min}$; faixa de temperatura 50-320 ${ }^{\circ} \mathrm{C}$, com razão de aquecimento $5{ }^{\circ} \mathrm{C} / \mathrm{min}$.

\section{Densidade compactada}

A densidade compactada é um parâmetro físico, cuja faixa ideal de especificação, não encontrada oficialmente, deve ser determinada a partir de várias medidas em matéria-prima de fabricantes diversos. Os valores de densidade compactada encontrados para as matérias-primas GLIB estão apresentados na Tabela III. O valor para MP1 não foi determinado em função da pequena quantidade de matéria- prima recebida, insuficiente para o teste.

A amostra MP4 foi a que apresentou menor valor de densidade compactada em relação a MP2 e MP3, sugerindo ser uma matéria-prima com maior propensão a se compactar. Desta forma, o processo produtivo de mistura deve ser bem monitorado, com mais rigor, para que não haja problemas de heterogeneidade na fabricação dos comprimidos.

\section{Massa e área superficial específicas}

O pré-tratamento térmico empregado nas amostras teve por objetivo retirar umidade ou traços residuais de vapor ou qualquer outro solvente que pudessem ocupar algum espaço. Os resultados de massa e área superficial específicas estão apresentados na Tabela IV.

Os resultados de massa específica encontrados para as matérias-primas MP2, MP3 e MP4 foram coincidentes e acima do valor encontrado para MP1. Todas as matérias-primas, exceto MP1, seguiram a isoterma de BET apresentando linearidade segundo o modelo proposto por Brunauer, Emmet e Teller (1938) para cálculo da ASE. Este fato sugere que a matéria-prima MP1 possui poros de maior tamanho (macroporos, ou seja, poros > $1000 \AA$ ) conforme visualizado pela MEV. Medidas para este tamanho de poro não se enquadram à faixa de aplicação da isoterma de BET, a qual somente é valida para a determinação de microporos (<20 $\AA$ ) e mesoporos (entre $20 \AA$ e $500 \AA ̊)$ (Lowel, Shields, 1991; Leon y Leon, 1997). Além disso, o valor negativo encontrado para a referida matériaprima também indica que o gás selecionado não foi o mais adequado para a determinação de ASE de materiais com esta característica, sendo necessário, portanto, testar, por exemplo, o método da intrusão por mercúrio, mais adequado para materiais com macroporos.

Os resultados de ASE variam inversamente com o tamanho médio de partícula das matérias-primas, pois, à medida que ASE aumenta, o valor do tamanho médio de partícula diminui.

Na Farmacopéia Brasileira terceira edição (1977), a medida de ASE era preconizada para GLIB matéria-prima

TABELA II - Valores de absorvância ${ }^{a}$ obtidos para as soluções GLIB SQRFB e matérias-primas por espectrofotometria na região do ultravioleta.

\begin{tabular}{lcccccccccc}
\hline Solução & \multicolumn{2}{c}{ GLIB SQRFB } & \multicolumn{2}{c}{ MP1 } & \multicolumn{2}{c}{ MP1 } & \multicolumn{2}{c}{ MP3 } & \multicolumn{2}{c}{ MP4 } \\
$1(\mathrm{~nm})$ & 275 & 300 & 275 & 300 & 275 & 300 & 275 & 300 & 275 & 300 \\
\hline Absorvância $^{b}$ & 0,294 & 0,634 & 0,288 & 0,632 & 0,287 & 0,630 & 0,285 & 0,628 & 0,284 & 0,624 \\
& 0,287 & 0,629 & 0,289 & 0,633 & 0,288 & 0,631 & 0,290 & 0,629 & 0,284 & 0,626 \\
& 0,288 & 0,632 & 0,291 & 0,634 & 0,289 & 0,632 & 0,287 & 0,631 & 0,285 & 0,627 \\
\hline
\end{tabular}

${ }^{\mathrm{a}}$ concentração $0,1 \mathrm{mg} / \mathrm{mL}$ em metanol acidificado; ${ }^{\mathrm{b}}$ triplicata. 


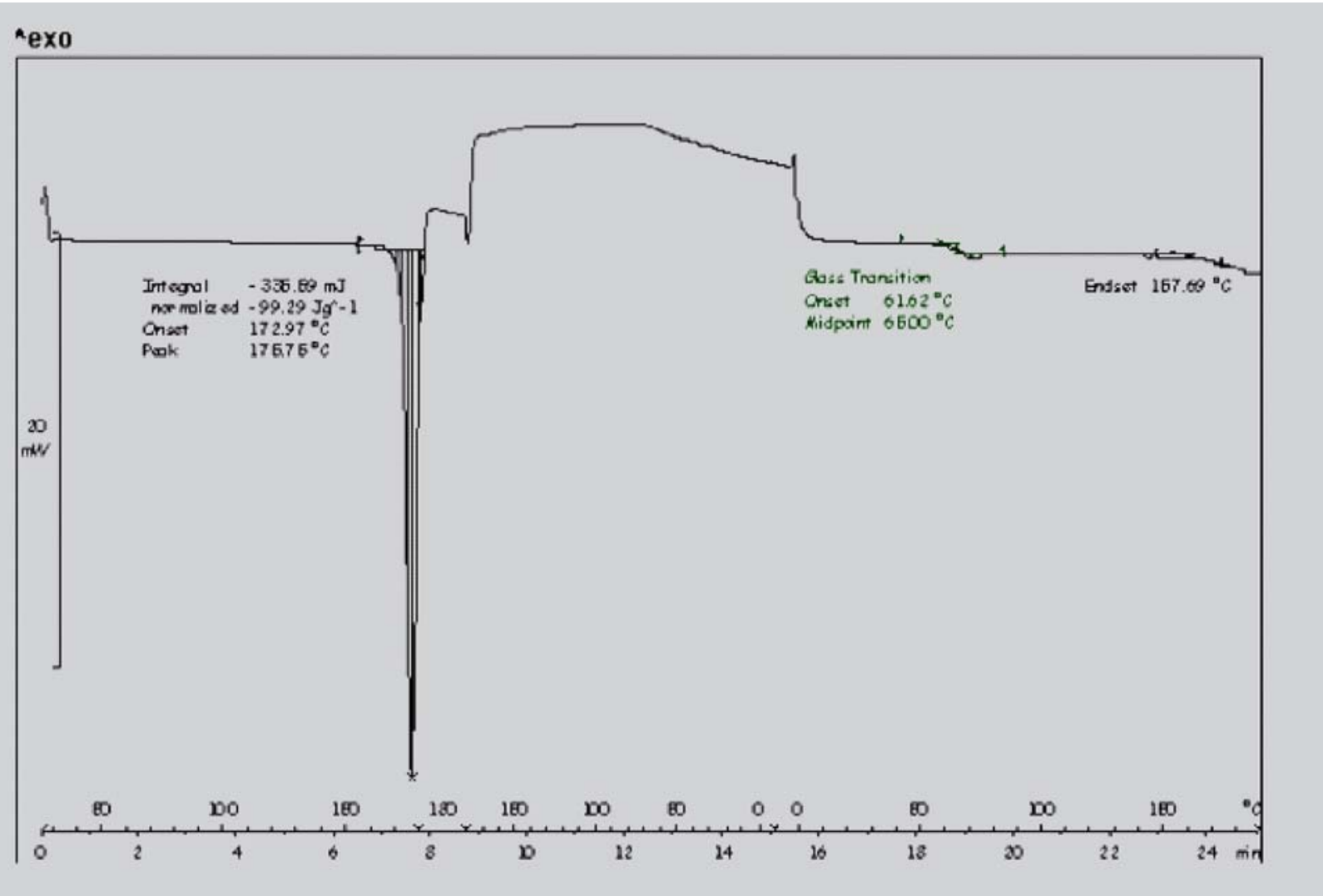

FIGURA 5 - Curva DSC de GLIB matéria-prima MP2. Condições: cadinho de alumínio $40 \mathrm{~mL}$ com tampa perfurada; pressão atmosférica com fluxo do gás nitrogênio $50 \mathrm{~mL} / \mathrm{min}$; faixa de temperatura $25-180^{\circ} \mathrm{C}$ com razão de aquecimento $20^{\circ} \mathrm{C} / \mathrm{min}$; isoterma $180^{\circ} \mathrm{C}$ por $1 \mathrm{~min}$; faixa de temperatura $180^{\circ} \mathrm{C}$ até $10^{\circ} \mathrm{C}$ com razão de resfriamento de $30^{\circ} \mathrm{C} / \mathrm{min}$; faixa de temperatura -10 até $190^{\circ} \mathrm{C}$ com razão de aquecimento de $20^{\circ} \mathrm{C} / \mathrm{min}$.

(especificação entre 1,0 e $2,0 \mathrm{~m}^{2} / \mathrm{g}$ ), porém, atualmente o teste não é mais exigido pela Farmacopéia Brasileira, quarta edição (2001). As matérias-primas MP2 e MP3, provenientes do mesmo fabricante, estão de acordo com tal especificação, enquanto que, MP4, com menor tamanho de partícula, apresentou valor superior. Com estes resultados, sugere-se uma revisão destes limites, pois, elevada ASE é útil para fármacos de baixa solubilidade como a GLIB. O valor de especificação para ASE, de não menos que $2,0 \mathrm{~m}^{2} / \mathrm{g}$ é sugerido para inclusão na monografia de GLIB matéria-prima para a próxima edição da Farmacopéia Brasileira.

\section{Difração de raios $X$}

Os valores, em porcentagem de intensidade relativa (I) e respectivas distâncias interplanares (d) obtidos para o cartão JCPDS (padrão) e matérias-primas MP1, MP2, MP3 e MP4 estão apresentados na Tabela V. A Figura 7 ilustra o difratograma de GLIB matéria-prima MP4, representativo das demais. Os sinais característicos de estado amorfo, encontrados na região de ângulo $0^{\circ}$ e $10^{\circ}$, não são aparentes nos difratogramas da GLIB. Caso existam, a proporção de GLIB amorfa pode ser inferior a $1 \%$ e a sua detecção não é possível neste equipamento. A presença de inúmeros picos ao longo do difratograma indica que as amostras apresentam estrutura essencialmente cristalina.

As matérias-primas apresentam valores bastante próximos de distâncias interplanares $(d)$ em 100\% de intensidade relativa (I), em relação ao padrão (cartão JCPDS). Nem todos os outros valores de distâncias interplanares e intensidades relativas são coincidentes mas apresentam-se próximos. Embora estas pequenas diferenças encontradas possam ser indicativas de arranjos cristalinos diferenciados entre as matérias-primas, a presença de polimorfos não foi confirmada na análise térmica (DSC) realizada.

A suspensão das matérias-primas em detergente é necessária para a boa dispersão de GLIB e obtenção de análises de distribuição granulométrica com boa reprodutibilidade. A otimização da velocidade de agitação da amostra no equipamento e a utilização de um agente tensoativo evitaram a formação de agregados ou aglomerados fracos que pudessem ser interpretados como partícu- 


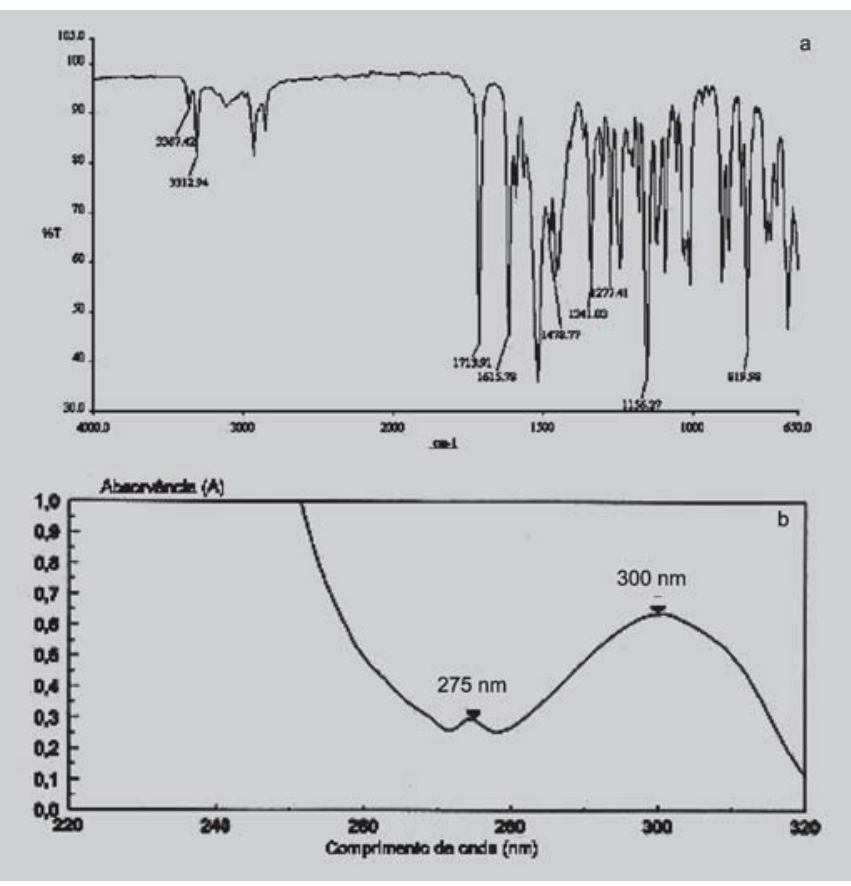

FIGURA 6 - Espectros no infravermelho (a, KBr) e no ultravioleta (b, 0,1 mg/L em solução metanólica acidificada, máximos de absorção em $275 \mathrm{~nm}$ e $300 \mathrm{~nm}$ ) de GLIB, representativos das matérias-primas MP1-MP4 e em concordância com os respectivos espectros de GLIB SQRFB.

TABELA III - Resultados ${ }^{a}$ de valores de densidade compactada encontrados para as matérias-primas GLIB.

\begin{tabular}{lcccc}
\hline Matéria-prima & MP1 & MP2 & MP3 & MP4 \\
\hline Densidade & ND & 0,517 & 0,576 & 0,333 \\
compactada $(\mathrm{g} / \mathrm{mL})$ & ND & 0,517 & 0,576 & 0,339 \\
Média $(\mathrm{g} / \mathrm{mL})$ & ND & 0,517 & 0,576 & 0,336 \\
\hline
\end{tabular}

${ }^{a}$ duplicata; ND, não determinado

TABELA IV - Resultados ${ }^{\mathrm{a}}$ de massa específica e área superficial específica obtidos para GLIB matérias-primas.

\begin{tabular}{lcc}
\hline Matéria-prima & $\begin{array}{c}\text { Massa } \\
\text { específica/ME } \\
\left(\mathrm{g} / \mathrm{cm}^{3}\right)\end{array}$ & $\begin{array}{c}\text { Área superficial } \\
\text { específica/ASE } \\
\left(\mathrm{m}^{2} / \mathrm{g}\right)\end{array}$ \\
\hline MP1 & 1,39 & $-0,04$ \\
MP2 & 1,44 & 1,25 \\
MP3 & 1,44 & 1,47 \\
MP4 & 1,44 & 2,87 \\
\hline
\end{tabular}

${ }^{a}$ média de duas determinações las individuais de tamanho maior. Ainda assim, após um determinado tempo de análise, forças de repulsão, parcialmente superadas pelas forças de atração, levaram à formação de aglomerados nas amostras, com exceção da matéria-prima MP1. Nesta última, os cristais grandes e aparentemente mais definidos e sua menor superfície de contato explicam este comportamento.

\section{Análise de distribuição granulométrica}

Na monografia de GLIB matéria-prima da terceira edição da Farmacopéia Brasileira (1977) preconiza que, pelo menos $90 \%$ das partículas devem estar abaixo de 50 $\mathrm{mm}$ e, pelo menos $98 \%$ das partículas, abaixo de $100 \mathrm{~mm}$. A distribuição de tamanho de partículas pode ser representada pelo valor mais freqüente (moda) e percentis 90 (P90), 98 (P98) e 100 (P100) (partículas com tamanho menor que $90 \%, 98 \%$ e $100 \%$ do total, respectivamente) (Tabela VI). Todas as matérias-primas apresentaram 90\% e $98 \%$ de suas partículas com tamanho inferior a $50 \mathrm{~mm}$ e $100 \mathrm{~mm}$, respectivamente, à exceção de MP1. As matérias-primas MP2 e MP3 apresentaram valores de tamanho médio, moda e P90 muito próximos entre si. Embora sejam matérias-primas provenientes do mesmo fabricante, existem $10 \%$ de partículas do total das amostras com tamanhos diferenciados impactando em valores bastante distintos em P100. A matéria-prima MP4 foi a que mais se distinguiu das demais, possuindo menor tamanho médio de partícula.

Os gráficos de distribuição granulométrica de GLIB matérias-primas MP1, MP2, MP3 e MP4 estão apresentados nas Figuras 8 a,b,c,d, respectivamente.

Considerando que a forma e o tamanho das partículas são indicadores de reprodutibilidade em um processo de síntese, bem como, fator importante na solubilidade e, conseqüentemente na dissolução e biodisponibilidade de uma forma farmacêutica, sugerem-se como especificações de tamanho de partícula, que $90 \%$ das partículas (P90) sejam menores que $10 \mathrm{~mm}$ e $100 \%$ das partículas (P100) sejam menores que $90 \mathrm{~mm}$, para inclusão na monografia GLIB matéria-prima da Farmacopéia Brasileira, próxima edição.

\section{CONCLUSÕES}

As diversas matérias-primas GLIB foram analisadas por técnicas que permitem a sua caracterização. A análise térmica por DSC é uma importante ferramenta no desenvolvimento e controle de qualidade de fármacos que apresentam polimorfismo. As matérias-primas GLIB disponíveis no mercado local brasileiro não apresentaram 
TABELA V - Resultados obtidos por difração de raios X (distâncias interplanares, $d$, e porcentagem de intensidade relativa, I) de GLIB matérias-primas MP1, MP2, MP3 e MP4 em comparação com o padrão (cartão JCPDS).

\begin{tabular}{lccccccccc}
\hline \multicolumn{2}{c}{ Padrão } & \multicolumn{2}{c}{ MP1 } & \multicolumn{2}{c}{ MP2 } & \multicolumn{2}{c}{ MP3 } & \multicolumn{2}{c}{ MP4 } \\
$d(\mathrm{~A})$ & $I(\%)$ & $d(\mathrm{~A})$ & $I(\%)$ & $d(\mathrm{~A})$ & $I(\%)$ & $d(\mathrm{~A})$ & $I(\%)$ & $d(\mathrm{~A})$ & $I(\%)$ \\
\hline 4,6733 & 100 & 4,6771 & 100 & 4,6795 & 100 & 4,6831 & 100 & 4,6778 & 100 \\
4,5562 & 73 & 4,2289 & 74 & 4,2290 & 71 & 4,2320 & 80 & 4,2271 & 66 \\
7,5472 & 62 & 3,8348 & 58 & 3,8347 & 58 & 3,8374 & 56 & 7,5525 & 56 \\
4,2262 & 56 & 7,5573 & 53 & 7,5559 & 49 & 4,5649 & 53 & 4,5619 & 54 \\
8,1240 & 42 & 4,5617 & 51 & 4,5624 & 42 & 7,5678 & 48 & 3,8349 & 48 \\
4,2732 & 36 & 3,8809 & 33 & 2,9441 & 36 & 3,8817 & 39 & 3,8854 & 34 \\
\hline
\end{tabular}

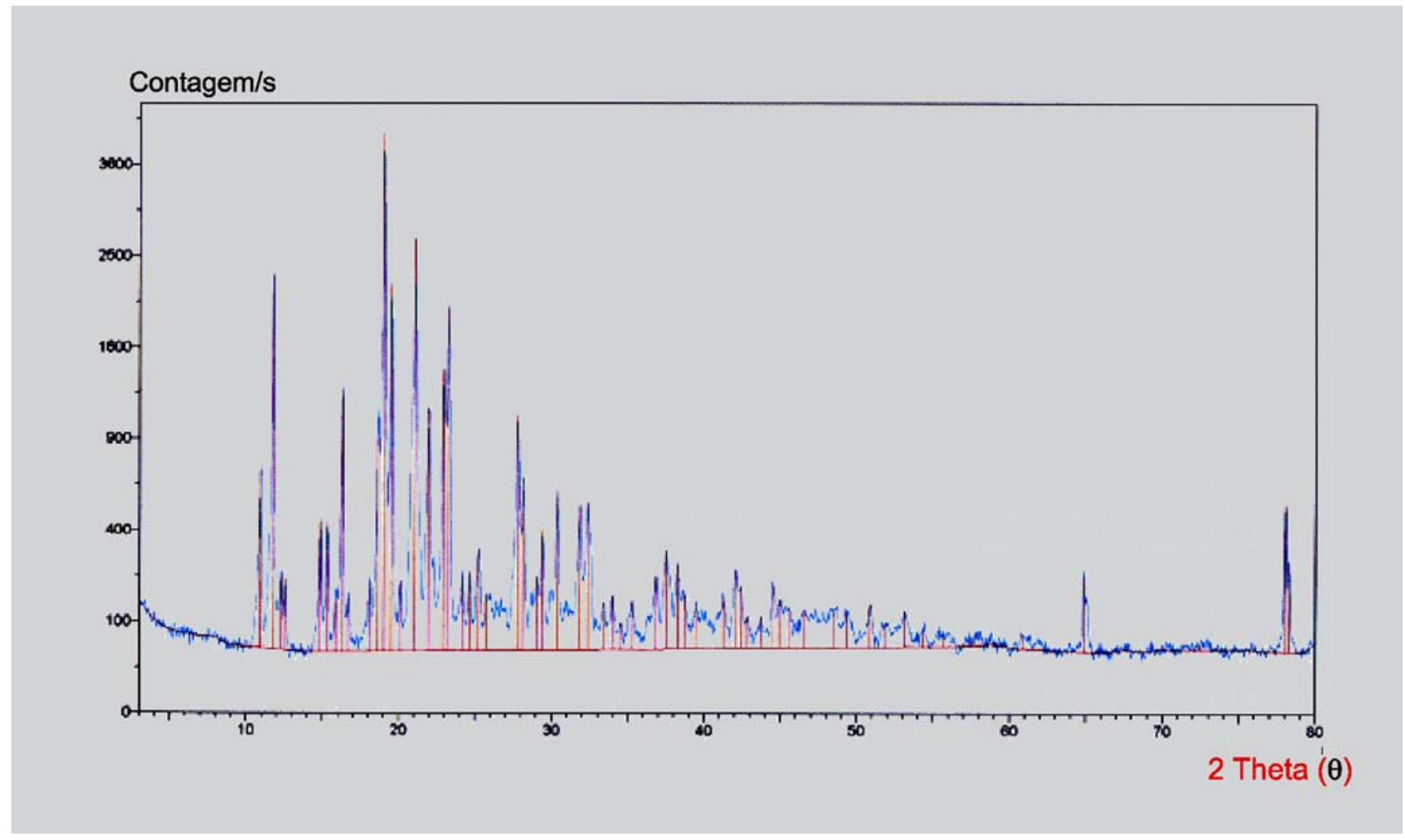

FIGURA 7 - Difratograma de raios X de GLIB matéria-prima MP4. Condições: sistema monocromador em cristal de grafite, radiação $\mathrm{Cu}-\mathrm{Ka}, 11,54186 \mathrm{~nm}$, voltagem e corrente $40 \mathrm{kV}$ e $20 \mathrm{~mA}$, respectivamente, varredura de raios $\mathrm{X}$ de ângulo aberto $2 \mathrm{q}$ entre $0-80^{\circ}$.

polimorfismo, apesar de relatos na literatura.

Algumas especificações complementares podem ser indicadas para inclusão na monografia de GLIB matériaprima como sugestão para a próxima edição da Farmacopéia Brasileira. Sugerem-se que os testes distribuição granulométrica e área superficial específica, devido à sua importância na caracterização de fármacos de baixa solubilidade, sejam novamente incluídos na monografia de GLIB matéria-prima com reavaliação e proposta de novos critérios de especificação.

\section{AGRADECIMENTOS}

Ao Departamento de Metalurgia (Escola de Engenharia/UFMG, Belo Horizonte) pela realização de micrografias eletrônicas de varredura, análises de densidade e área superficial específicas e difração de raios X; ao Laboratório Associado Micronal e Instituto de Pesquisas Tecnológicas (LAMI, São Paulo) pelas análises granulométricas e térmicas e à FAPEMIG, pelo apoio financeiro. À Professora Maria Irene Yoshida pela revisão relativa à análise térmica. 

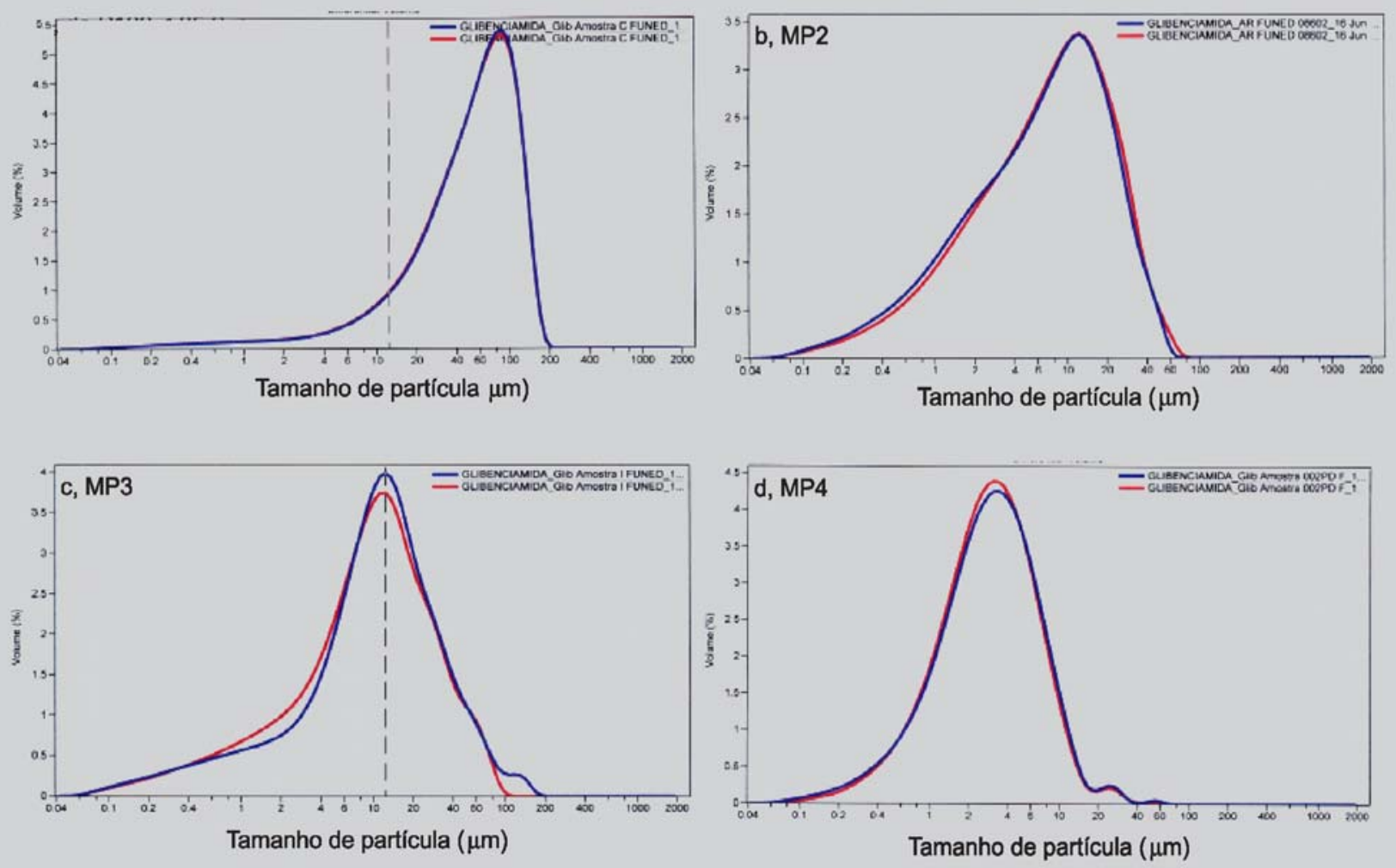

FIGURA 8 - Gráficos da distribuição granulométrica (duplicata) de GLIB matérias-primas MP1 (a), MP2 (b), MP3 (c) e MP4 (d).

TABELA VI - Resultados ${ }^{\mathrm{a}}$ da distribuição de tamanho de partícula (duplicata) para GLIB matérias-primas MP1, MP2, MP3 e MP4.

\begin{tabular}{lccccc}
\hline $\begin{array}{l}\text { Matéria- } \\
\text { prima }\end{array}$ & Média & Moda & P90 & P98 & P100 \\
\hline MP1 & 60,73 & 87,90 & 117,60 & 149,3 & 234,0 \\
& 60,04 & 87,90 & 116,90 & 148,1 & 234,0 \\
MP2 & 10,25 & 12,40 & 24,21 & 40,51 & 76,42 \\
& 10,91 & 12,40 & 25,56 & 42,84 & 92,09 \\
MP3 & 16,83 & 12,40 & 38,18 & 81,38 & 213,20 \\
& 14,52 & 12,40 & 34,45 & 63,33 & 121,80 \\
MP4 & 2,91 & 3,36 & 8,21 & 15,51 & 83,89 \\
& 3,89 & 3,06 & 7,83 & 14,23 & 76,12 \\
\hline
\end{tabular}

\section{ABSTRACT}

\section{Characterization of the hypoglycemic drug glibenclamide}

Glibenclamide (GLIB) or glyburide, a second-generation hypoglycemic agent is orally used in the form of tablets for the treatment of diabetes mellitus. Bulk GLIB has a low aqueous solubility and it may yield low drug release in the dissolution test, causing variabilility in the treatment. This work evaluates the bulk GLIB features, which may influence drug release profile, hence, bioavailability, by means of techniques such as nitrogen sorption analysis, laser diffraction, thermal analysis, IV/UV spectroscopy and $X$-ray analysis.

UNITERMS: Hypoglycemic agent. Glibenclamidel characterization.

\section{REFERÊNCIAS}

AHFS drug information 2000. Bethesda: American Society of Health System Pharmacist, 2000, p.2850-2856.

BABU, R.J.; PANDIT, J.K. Enhancement of dissolution rate and hypoglycemic activity of glibenclamide with bcyclodextrin. S.T.P. Pharma Sci., v.5, p.196-201, 1995. 
BRASIL. Ministério da Saúde. Anvisa aprova primeiros genéricos para tratamento da diabetes. Brasília, 2001a. Disponível em: $<\mathrm{http}: / /$ portalweb02.saude.gov.br/saude/ aplicacoes/noticias/noticias_detalhe.cfm? co_seq noticia $=427>$. Acesso em: 19 set. 2002.

BRASIL. Ministério da Saúde. Durante a Campanha Nacional de Detecção de Diabetes foram examinados mais de 20 milhões de pessoas. Brasília, 2001b. Disponível em: $<\mathrm{http}: / /$ portalweb02.saude.gov.br/saude/ aplicacoes/noticias/noticias_detalhe.cfm? co_seq_noticia $=415>$. Acesso em: 19 set. 2002

BRASIL. Ministério da Saúde. Saúde aprova genérico para tratamento de gota e diabetes. Brasília, 2001b. Disponível em: <http://portalweb02.saúde.gov.br/saúde/ aplicacoes/noticias/noticias_detalhe.cfm? co_seq_noticia $=458>$. Acesso em: 20 set. 2002.

BRITISH pharmacopeia. London: Her Majesty's Stationery Office, 2003a. v.1, p.871-872.

BRITISH pharmacopeia. London: Her Majesty's Stationery Office, 2003b. v.3, p.2358-2359.

BRUNAUER, S.; EMMETT, P.H.; TELLER, E. Adsorption of gases in multimolecular layers. J. Am. Chem. Soc. v.60, p.309-319, 1938.

CLARKE, E.G.C., (Org.). Isolation and identification of drugs. 2.ed. London: Pharmaceutical Press, 1986. p.638639.

CLAS, S-D; DALTON, C.R; HANCOCK, B.C. Differential scanning calorimetry: application in drug development Pharm. Sci. Technol. Today, v.2, p.311-320, 1999.

EUROPEAN pharmacopeia. Strasbourg: European Department for Quality of Medicines, 1997. p.898.

FARMACOPEA de los Estados Unidos Mexicanos. 7.ed. México: Secretaría de Salud, 2000. p.2517-2518.

FARMACOPÉIA brasileira. 3.ed. São Paulo: Andrei, 1977, p.491-492.

FARMACOPÉIA brasileira. 4.ed. São Paulo: Atheneu, 1988. pt. 1. p.V.2.2.

FARMACOPÉIA brasileira. 4.ed. São Paulo: Atheneu, 2001, pt. 2, p.153-153.1.
FLORENCE, A.T., ATTWOOD, D. Princípios físicoquímicos em farmácia. São Paulo: Editora da Universidade de São Paulo, 2003. p.29-66.

GHOSH, L.K.; THAKUR, R. S.; SHARMA, N. C.; GHOSH, N. C.; GUPTA, B.K. Development and evaluation of an ideal formulation of glibenclamide by solid dispersion techniques. Boll. Chim. Farm., v.137, p.26-29, 1998.

HASSAN, M.A; NAJIB, N.M.; SULEIMAN, M.S. Characterization of glibenclamide glassy state. Int. J. Pharm. v.67, p.131-137, 1991.

JAPANESE Pharmacopoeia. 14.Ed. 2001. pt. 2. Disponível em: $<$ http://jpdh.nihs.go.jp/jp14e> Acesso em: 10 jul. 2003.

KASIM, N.A.; WHITEHOUSE, M.; RAMACHANDRAN, C.; BERMEJO, M.; LENNERNAS, H.; HUSSAIN, JUNGINGER, H.E.; STAVCHANSKY, S.A; MIDHA, K.K.; SHAH, V.P.; AMIDON, G.L. Molecular properties of WHO essential drugs and provisional biopharmaceutical classification. Molec. Pharm., v.1, p.85-96, 2004.

LEON y LEON, C.A.; THOMAS, M.A. Recent advances in the interpretation of mercury porosimetry data. GIT Lab. J. Int. Ed., v. 1, p.101-104, 1997.

LOWEL, S.; SHIELDS, J.E. Powder surface area and porosity. London: Chapman \& Hall. 1991. 205 p.

MERCK index: an encyclopedia of chemicals, drugs and biologicals. 14. ed. Whitehouse Station: Merck Research Laboratories, 2006. p.773.

NERY, C.G.C.; PIANETTI, G.A.; PIRES, M.A.S.; MOREIRA-CAMPOS, L.M.; VIANNA-SOARES, C.D. Rev. Bras. Ciênc. Farm., v.43, n.3, p.413-419, 2007.

PANAGOPOULOU-KAPLANI, A.; MALAMATARIS, S. Preparation and characterisation of a new insoluble polymorphic form of glibenclamide. Int.J. Pharm. v.195, p.239-246, 2000,

SULEIMAN, M.S.; NAJIB, N.M. Isolation and physicochemical characterization of solid forms. Int. J. Pharm. v.50, p.103-109, 1989. 
TAKLA, P.G. Glibenclamide. Anal. Profiles Drug Subst., v.10, p.338-355, 1981.

UNITED States Pharmacopeia. The National Formulary. 29.ed. Rockville: United States Pharmacopeial Convention, 2005. p.1010-1011.
YOSHIHASHI, Y.; KITANO, H.; YONEMOCHI, E.; TERADA, K. Quantitative correlation between initial dissolution rate and heat of fusion of drug substance. Int. J. Pharm., v.204, p.1-6, 2000.

Recebido para publicação em 02 de fevereiro de 2007. Aceito para publicação em 22 de agosto de 2007. 